\title{
Nuclear pasta phases within the quark-meson coupling model
}

\author{
Guilherme Grams, ${ }^{1}$ Alexandre M. Santos, ${ }^{1}$ Prafulla K. Panda, ${ }^{2}$ Constança Providência, ${ }^{3}$ and Débora P. Menezes ${ }^{1}$ \\ ${ }^{1}$ Departamento de Física, CFM, Universidade Federal de Santa Catarina, Florianópolis, Brazil \\ ${ }^{2}$ Department of Physics, Utkal University, Bhubaneswar-751 004, India \\ ${ }^{3}$ CFisUC, Department of Physics, University of Coimbra, P-3004-516 Coimbra, Portugal
}

(Received 4 November 2016; revised manuscript received 21 March 2017; published 19 May 2017)

\begin{abstract}
In this work, the low-density regions of nuclear and neutron star matter are studied. The search for the existence of nuclear pasta phases in this region is performed within the context of the quark-meson coupling (QMC) model, which incorporates quark degrees of freedom. Fixed proton fractions are considered, as well as nuclear matter in $\beta$ equilibrium at zero temperature. We discuss the recent attempts to better understand the surface energy in the coexistence phases regime and we present results that show the existence of the pasta phases subject to some choices of the surface energy coefficient. We also analyze the influence of the nuclear pasta on some neutron star properties. The equation of state containing the pasta phase will be part of a complete grid for future use in supernova simulations.
\end{abstract}

DOI: 10.1103/PhysRevC.95.055807

\section{INTRODUCTION}

At very low nuclear matter density, a competition between the strong and the electromagnetic interactions takes place [1,2], leading to a configuration in which its free energy per particle may be lower than that corresponding to the homogeneous phase at the same density. The so-called pasta phases are therefore the preferred shapes of some systems at these densities [3-6]. These structures look like droplets, bubbles, rods, tubes, and slabs [3] and are expected to exist $[7,8]$ both in the crust of neutron stars (zero temperature, very low proton fraction, matter in $\beta$ equilibrium) and in supernova (finite temperature, proton fraction around 0.3).

From analysis of these glitches, the authors of Ref. [9] have related the fraction of the moment of inertia contained in the crust of the Vela pulsar with the mass and the radius of the neutron star and the pressure and density at the crust-core interface. From realistic equations of state (EoS), they have obtained an expected range of values for the pressure at the inner edge of the crust and therefore also a relation between the radius and mass of the pulsar. This work shows the importance of understanding the exact density limits of the pasta phases and its consequences on the choice of appropriate equations of state. More recently, the existence of the pasta phase in the neutron star crust was shown to considerably alter the neutrino mean-free paths and its diffusion coefficients as compared with the homogeneous matter results. The consequent differences in neutrino opacities certainly influence the Kelvin-Helmholtz phase of the star evolution [10,11].

On the other hand, due to well-known observational difficulties, simulations of core-collapse supernova have played an important role in the study of supernovae explosions and the evolution of their possible remnants. Hence, obtaining appropriate equations of state $(\mathrm{EoS})$ for core-collapse supernova simulations has been a very challenging task. For this class of EoS, one needs a grid of thermodynamic quantities with densities ranging from $10^{5}$ to more than $10^{15} \mathrm{~g} \mathrm{~cm}^{-3}$, proton fractions up to about 0.6 , and temperatures varying from zero to more than $100 \mathrm{MeV}$. So far, in almost all models used for the obtainment of a complete grid with the aim of being tested in supernova simulations, inhomogeneous matter believed to be present at low densities has been considered only with the inclusion of clusters [12-16]. The EoS of Lattimmer and Swesty [17] takes into account the presence of bubbles besides the clusters and allows for the existence of different shapes in a phenomenological construction. However, according to Refs. [18,19], the pasta phase may form $10-20 \%$ of the mass of the supernova core, and therefore its role should not be disregarded. The pasta phase has been studied in the context of several models [1,19-22] and all of them predict its existence under the conditions expected to be found in the inner crust of compact objects, although the profiles show that it varies in many aspects $[8,20]$.

The quark-meson coupling (QMC) model [23-25] describes nuclear matter as a system of nonoverlapping MIT-like bags, interacting with each other by interchanging meson fields. Hence, it contains more fundamental degrees of freedom than the usual quantum hadrodynamic models, so far used in the study of the pasta phases $[1,10,20,26]$. With the aim of constructing a complete grid for supernova simulations, a preliminary work at zero temperature, $\rho=10^{14}-10^{16} \mathrm{~g} \mathrm{~cm}^{-3}$ and $Y_{p}=0-0.65$, was done [27] where a comparison with other models revealed that the QMC is a promising model.

In the present work, we study the possible existence of the pasta structures within the QMC model at zero temperature and its dependence on the surface energy coefficient. The work is organized as follows: In Sec. II, the QMC model is briefly reviewed and the method of the coexisting phases used to build the pasta phase is presented in Sec. III, where a detailed study of the surface tension coefficient is performed. In Sec. IV, we present our results and draw the conclusions. In the last section, we make some final remarks.

\section{THE QUARK-MESON COUPLING MODEL}

In the QMC model, the nucleon in nuclear medium is assumed to be a static spherical MIT bag in which quarks interact with the scalar $(\sigma)$ and vector $(\omega, \rho)$ fields, and those are treated as classical fields in the mean field approximation 
(MFA) [23]. The quark field, $\psi_{q_{N}}$, inside the bag then satisfies the equation of motion:

$$
\begin{aligned}
{\left[i \not \partial-\left(m_{q}^{0}-g_{\sigma}^{q}\right)-g_{\omega}^{q} \omega \gamma^{0}+\frac{1}{2} g_{\rho}^{q} \tau_{z} \rho_{03} \gamma^{0}\right] \psi_{q_{N}}(x) } & =0, \\
q & =u, d,
\end{aligned}
$$

where $m_{q}^{0}$ is the current quark mass and $g_{\sigma}^{q}, g_{\omega}^{q}$, and $g_{\rho}^{q}$ denote the quark-meson coupling constants. The normalized ground state for a quark in the bag is given by

$$
\begin{aligned}
\psi_{q_{N}}(\mathbf{r}, t)= & \mathcal{N}_{q_{N}} \exp \left(-i \epsilon_{q_{N}} t / R_{N}\right) \\
& \times\left(\begin{array}{c}
j_{0_{N}}\left(x_{q_{N}} r / R_{N}\right) \\
i \beta_{q_{N}} \vec{\sigma} \cdot \hat{r} j_{1_{N}}\left(x_{q_{N}} r / R_{N}\right)
\end{array}\right) \frac{\chi_{q}}{\sqrt{4 \pi}},
\end{aligned}
$$

where

$$
\epsilon_{q_{N}}=\Omega_{q_{N}}+R_{N}\left(g_{\omega}^{q} \omega+\frac{1}{2} g_{\rho}^{q} \tau_{z} \rho_{03}\right)
$$

and

$$
\beta_{q_{N}}=\sqrt{\frac{\Omega_{q_{N}}-R_{N} m_{q}^{*}}{\Omega_{q_{N}}+R_{N} m_{q}^{*}}},
$$

with the normalization factor given by

$$
\mathcal{N}_{q_{N}}^{-2}=2 R_{N}^{3} j_{0}^{2}\left(x_{q}\right)\left[\Omega_{q}\left(\Omega_{q}-1\right)+R_{N} m_{q}^{*} / 2\right] / x_{q}^{2},
$$

where $\Omega_{q_{N}} \equiv \sqrt{x_{q_{N}}^{2}+\left(R_{N} m_{q}^{*}\right)^{2}}, m_{q}^{*}=m_{q}^{0}-g_{\sigma}^{q} \sigma, R_{N}$ is the bag radius of nucleon $N$, and $\chi_{q}$ is the quark spinor. The bag eigenvalue for nucleon $N, x_{q_{N}}$, is determined by the boundary condition at the bag surface

$$
j_{0_{N}}\left(x_{q_{N}}\right)=\beta_{q_{N}} j_{1_{N}}\left(x_{q_{N}}\right) .
$$

The energy of a static bag describing nucleon $N$ consisting of three quarks in ground state is expressed as

$$
E_{N}^{\mathrm{bag}}=\sum_{q} n_{q} \frac{\Omega_{q_{N}}}{R_{N}}-\frac{Z_{N}}{R_{N}}+\frac{4}{3} \pi R_{N}^{3} B_{N},
$$

where $Z_{N}$ is a parameter which accounts for zero-point motion of nucleon $N$ and $B_{N}$ is the bag constant. The set of parameters used in the present work is determined by enforcing stability of the nucleon (here, the "bag"), much like in Ref. [28], so there is a single value for proton and neutron masses. The effective mass of a nucleon bag at rest is taken to be $M_{N}^{*}=E_{N}^{\mathrm{bag}}$.

The equilibrium condition for the bag is obtained by minimizing the effective mass, $M_{N}^{*}$, with respect to the bag radius

$$
\frac{d M_{N}^{*}}{d R_{N}^{*}}=0, \quad N=p, n .
$$

By fixing the bag radius $R_{N}=0.6 \mathrm{fm}$ and the bare nucleon mass $M=939 \mathrm{MeV}$, the unknowns $Z_{N}=4.0050668$ and $B_{N}^{1 / 4}=210.85 \mathrm{MeV}$ are then obtained. Furthermore, the desired values of $B / A \equiv \epsilon / \rho-M=-16.45 \mathrm{MeV}$ at saturation $n=n_{0}=0.15 \mathrm{fm}^{-3}$ are achieved by setting $g_{\sigma}^{q}=$ 5.9810, $g_{\omega}=8.9817, g_{\rho}=8.6510$, where $g_{\omega}=3 g_{\omega}^{q}$ and $g_{\rho}=g_{\rho}^{q}$. The meson masses are $m_{\sigma}=550 \mathrm{MeV}, m_{\omega}=$ $783 \mathrm{MeV}$, and $m_{\rho}=770 \mathrm{MeV}$. With this parameterization, some of the bulk properties at saturation density, such as the
TABLE I. Nuclear matter bulk properties obtained with the QMC model. All quantities are taken at saturation.

\begin{tabular}{lcccccccc}
\hline \hline Model & $\begin{array}{c}B / A \\
(\mathrm{MeV})\end{array}$ & $\begin{array}{c}n_{0} \\
\left(\mathrm{fm}^{-3}\right)\end{array}$ & $\Lambda_{v}$ & $g_{\rho}$ & $M^{*} / M$ & $\begin{array}{c}J \\
(\mathrm{MeV})\end{array}$ & $\begin{array}{c}L_{0} \\
(\mathrm{MeV})\end{array}$ & $\begin{array}{c}\mathrm{K} \\
(\mathrm{MeV})\end{array}$ \\
\hline QMC & -16.4 & 0.15 & 0.0 & 8.6510 & 0.77 & 34.5 & 90 & 295 \\
QMC $\omega \rho$ & -16.4 & 0.15 & 0.03 & 9.0078 & 0.77 & 30.9 & 69 & 295 \\
\hline \hline
\end{tabular}

the compressibility, the symmetry energy, and the slope of the symmetry energy, are given in Table I. For a slightly different bag value, i.e., $B_{N}^{1 / 4}=211.033 \mathrm{MeV}$, the binding energy is $-15.7 \mathrm{MeV}$ and the symmetry energy and its slope become respectively 92.56 and $33.4 \mathrm{MeV}$. The results which we discuss next are very similar for both bag values and relevant parameter sets, i.e., the one just mentioned and the one shown in Table I. The properties at saturation of the QMC model, given in the first line of Table I, are within the accepted values (see Refs. [29,30], for instance), except for $L_{0}$ that presents a value which is already considered too large. However, $J$ and $L_{0}$ can be easily controlled by the inclusion of a $\omega \rho$ interaction, as discussed in Refs. [31-33]. As the value of this interaction gets larger, the the values of the symmetry energy and its slope become lower. We have next also included an $\omega \rho$ interaction strength that results in a symmetry energy equal to $22 \mathrm{MeV}$ at $0.1 \mathrm{fm}^{-3}$ with a consequent change in the $g_{\rho}$ coupling constant. The new values of the symmetry energy and its slope at saturation are also given in Table I. In this work, we study how much the choice of the parametrization affects the pasta phase structure and influences stellar matter. Other parametrizations are also possible. Of particular interest is the modified QMC model, where the parameters are adjusted so that the constituent quarks are confined to a flavor-independent potential where pionic and gluonic corrections are taken into account $[34,35]$. These studies will be performed in future investigations. Within the parametrization we have chosen, the total energy density of the nuclear matter reads

$$
\begin{aligned}
\varepsilon= & \frac{1}{2} m_{\sigma}^{2} \sigma+\frac{1}{2} m_{\omega}^{2} \omega_{0}^{2}+\frac{1}{2} m_{\rho}^{2} \rho_{03}^{2}+3 \Lambda_{v} g_{\omega}^{2} g_{\rho}^{2} \omega_{0}^{2} \rho_{03}^{2} \\
& +\sum_{N} \frac{1}{\pi^{2}} \int_{0}^{k_{N}} k^{2} d k\left[k^{2}+M_{N}^{* 2}\right]^{1 / 2},
\end{aligned}
$$

and the pressure is

$$
\begin{aligned}
p= & -\frac{1}{2} m_{\sigma}^{2} \sigma+\frac{1}{2} m_{\omega}^{2} \omega_{0}^{2}+\frac{1}{2} m_{\rho}^{2} \rho_{03}^{2}+\Lambda_{v} g_{\omega}^{2} g_{\rho}^{2} \omega_{0}^{2} \rho_{03}^{2} \\
& +\sum_{N}\left(\frac{1}{\left(3 \pi^{2}\right)}\right) \int_{0}^{k_{N}} k^{4} d k /\left[k^{2}+M_{N}^{* 2}\right]^{1 / 2} .
\end{aligned}
$$

The vector mean fields $\omega_{0}$ and $\rho_{03}$ are determined through

$$
\omega_{0}=\frac{g_{\omega}\left(n_{p}+n_{n}\right)}{m_{\omega}^{*^{2}}}, \quad \rho_{03}=\frac{g_{\rho}\left(n_{p}-n_{n}\right)}{2 m_{\rho}^{*^{2}}},
$$

where

$$
n_{B}=n_{p}+n_{n}=\sum_{N} \frac{k_{N}^{3}}{3 \pi^{2}}, \quad N=p, n,
$$


is the baryon density and $m_{\omega}^{*}, m_{\rho}^{*}$ are the effective masses of the meson fields given by $m_{\omega}^{*^{2}}=m_{\omega}^{2}+2 \Lambda_{v} g_{\omega}^{2} g_{\rho}^{2} \rho_{03}^{2}$ and $m_{\rho}^{*^{2}}=m_{\rho}^{2}+2 \Lambda_{v} g_{\omega}^{2} g_{\rho}^{2} \omega_{0}^{2}$.

Finally, the mean field $\sigma$ is fixed by imposing that

$$
\frac{\partial \varepsilon}{\partial \sigma}=0
$$

As mentioned in the introduction, our interest lies in matter at fixed proton fraction given by $Y_{p}=n_{p} / n_{B}$ as well as in stellar matter in $\beta$-equilibrium conditions, which for the system made up of protons, neutrons, and electrons is

$$
\mu_{p}=\mu_{n}-\mu_{e} .
$$

Charge neutrality requires that

$$
n_{p}=n_{e} .
$$

In this article, we work with the low-density regions of the neutron stars and in this region muons are not present.

\section{COEXISTING PHASES APPROXIMATION}

In this approximation, matter is organized in regions of lower density, generally with a neutron gas in the background and regions of higher density. For a given total density $n_{B}$ and proton fraction $Y_{p}$, the pasta structures are built with different geometrical forms. The forms are usually called sphere (bubble), cylinder (tube), and slab, in three, two, and one dimensions, respectively. This is achieved by calculating the density and the proton fraction of the pasta and of the background gas from the Gibbs conditions, that impose that both phases have the same pressure and proton and neutron chemical potentials, so that the following equations must be solved simultaneously:

$$
\begin{gathered}
P^{I}=P^{I I}, \\
\mu_{p}^{I}=\mu_{p}^{I I}, \\
\mu_{n}^{I}=\mu_{n}^{I I}, \\
n_{p}=n_{B} Y_{p}=f n_{p}^{I}+(1-f) n_{p}^{I I},
\end{gathered}
$$

where $I(I I)$ label the high- (low-) density phase, $n_{p}$ is the global proton density, and $f$ is the volume fraction of phase $I$,

$$
f=\frac{n_{B}-n_{B}^{I I}}{n_{B}^{I}-n_{B}^{I I}}
$$

If stellar matter is considered, the above equations are slightly altered in such a way that

$$
\begin{aligned}
& \mu_{n}^{I}=\mu_{n}^{I I}, \\
& \mu_{e}^{I}=\mu_{e}^{I I},
\end{aligned}
$$

and

$$
f\left(n_{p}^{I}-n_{e}^{I}\right)+(1-f)\left(n_{p}^{I I}-n_{e}^{I I}\right)=0,
$$

along with Eq. (16). Here, the density of electrons is no longer uniform as in the fixed proton fraction case. It appears as the solution of the above equation. After the lowest energy state is achieved, the energy obtained for hadronic matter is given by

$$
\varepsilon_{\text {matter }}=f \varepsilon^{I}+(1-f) \varepsilon^{I I}+\varepsilon_{e},
$$

to which the surface and Coulomb terms are added to account for the total energy density of the system, which becomes

$$
\varepsilon=\varepsilon_{\text {matter }}+\varepsilon_{\text {surf }}+\varepsilon_{\text {Coul }} .
$$

By minimizing the sum $\varepsilon_{\text {surf }}+\varepsilon_{\text {Coul }}$ with respect to the size of the droplet (bubble), rod (tube), or slab, we get [6] $\varepsilon_{\text {surf }}=$ $2 \varepsilon_{\text {Coul }}$, where

$$
\varepsilon_{\text {Coul }}=\frac{2 \alpha}{4^{2 / 3}}\left(e^{2} \pi \Phi\right)^{1 / 3}\left[\mathcal{S} D\left(n_{p}^{I}-n_{p}^{I I}\right)\right]^{2 / 3},
$$

where $\alpha=f$ for droplets, rods, and slabs, and $\alpha=1-f$ for tubes and bubbles. $\mathcal{S}$ is the surface tension discussed in the next subsection and $\Phi$ is given by

$$
\Phi=\left\{\begin{array}{cl}
\left(\frac{2-D \alpha^{1-2 / D}}{D-2}+\alpha\right) \frac{1}{D+2}, & D=1,3 \\
\frac{\alpha-1-\ln \alpha}{D+2}, & D=2
\end{array} .\right.
$$

As we are treating only the low-density region, we follow the parametrization prescription proposed in Ref. [36] for the effective nucleon mass as a nonlinear function of the $\sigma$ meson:

$$
M_{N}^{*}=M_{N}-g_{\sigma N}(\sigma) \sigma
$$

with

$$
g_{\sigma N}(\sigma)=\left(1+\frac{b}{2} \sigma+\frac{c}{3} \sigma^{2}\right) g_{\sigma N},
$$

where $g_{\sigma N}=3 g_{\sigma}^{q} S_{N}(0)=8.6157, b=-0.0007141$ $96 \mathrm{MeV}^{-1}$, and $c=9.84481 \times 10^{-8} \mathrm{MeV}^{-2}$. To obtain these parameters, we have plotted the exact effective mass at subsaturation density and fitted the curve with a polynomial expression. Our best fitting gives only the terms mentioned in Eq. (29) and one can see that the $c$ value is small, which means that a cubic term in $\sigma$ is only a correction that we have opted to keep in our calculation. Notice that these values are valid only for this specific parameterization.

Before we proceed to the discussion of the surface tension coefficient, it is important to point out that the coexistence phase (CP) method does not take into account the Coulomb interaction and finite-size effects in a self-consistent way. An alternative prescription within the compressible liquid drop (CLD) model incorporates these important effects by minimizing the total free energy, where surface and Coulomb terms are explicitly included [37] self-consistently. The resulting pressure and proton chemical potential equilibrium conditions are slightly different from the ones above. The differences between both prescriptions (CP and CLD) can be easily seen in Ref. [38] and the resulting pasta properties differ at very low densities [37,38], generally lower than $10^{-3} \mathrm{fm}^{-3}$ when the matching to the outer crust EoS is performed. As will be shown next, our calculation depends also on a free parameter, that is fitted according to accepted values of the surface tension.

\section{A. The surface tension coefficient}

When Gibbs's conditions are used in the coexistence phases approximation, the surface tension coefficient is always a 
tricky point, since the existence of the pasta phase depends on its value. Different considerations on this coefficient have been made in the literature $[1,6,20,26,39,40]$ and a choice generally employed is to fit the surface energy according to the Thomas-Fermi results. Since there are no Thomas-Fermi calculations for the QMC model to guide us, we have opted to use a method with more physical ingredients than simply choosing one specific value for $\mathcal{S}$, as done in many papers both for quark and hadronic matter. Some calculations based on nuclear mass formula fits produce results ranging from 1.06 to $1.34 \mathrm{MeV} \mathrm{fm}^{-2}$ [17] and these values have been used randomly. In the present work, to achieve a numerical value for the surface tension coefficient, the geometrical approach introduced in Ref. [41] is used next. In Ref. [42], this method was used to compute the surface tension in quark matter but recently it was also used to obtain the surface tension coefficient for hadronic matter [26]. The main ideas are also discussed next.

The surface tension coefficient, $\mathcal{S}$, which measures the energy per unit area necessary to create a planar interface between the two phases, is given by

$$
\mathcal{S}=\frac{a}{n_{g}} \sqrt{2 \varepsilon_{g}} \int_{n_{1}}^{n_{2}} \sqrt{\Delta \varepsilon} d n,
$$

where $n_{g}=\frac{n_{1}+n_{2}}{2}, \varepsilon_{g}=\frac{\varepsilon\left(n_{1}\right)+\varepsilon\left(n_{2}\right)}{2}, n_{1}$, and $n_{2}$ are the two coexistence baryonic density points and $\Delta \varepsilon=\varepsilon_{h m}-\varepsilon_{n h m}$ is the difference between the energy density of the homogeneous and the nonhomogeneous matter. The energy densities for both phases are obtained from Eqs. (9) and (24) and then fitted to a functional form given by $\varepsilon_{i}=\alpha_{i} n^{2}+\beta_{i} n+\gamma_{i}, i=h m, n h m$. Graphs showing this construction for hadronic matter can be found in Ref. [26] and we do not reproduce them here. In this geometrical approach, the width of the interface region and the magnitude of $\mathcal{S}$ are controlled by the adjustable parameter $a$ present in Eq. (30). In Ref. [42] the authors used $a=1 / m_{\sigma}=0.33 \mathrm{fm}$, where $m_{\sigma}=600 \mathrm{MeV}$ is the mass of the $\sigma$ meson, a natural scale for quark matter. As we are treating hadronic matter in the present work, our initial guess was $a=$ $1 / M_{N}=0.21 \mathrm{fm}$, where $M_{N}=939 \mathrm{MeV}$ is the nucleon mass. Another attempt followed the recipe used to find the surface tension of hadronic matter in Ref. [26] with an extended version of the Nambu-Jona-Lasino model, where $a=0.1 \mathrm{fm}$ was adopted to reproduce the value of the surface tension coefficient for the NL3 model [43] within a Thomas-Fermi calculation [39]. Our final choice was $a=0.023 \mathrm{fm}$, so that the value $\mathcal{S}=1.123 \mathrm{MeV} \mathrm{fm}^{-2}$ for $Y_{p}=0.5$ was reproduced as in Refs. $[26,39,40]$. Note that this value is the same for all values of the interaction strenght $\Lambda_{v}$ because it was chosen for symmetric nuclear matter, where the $\omega \rho$ interaction plays no role. In Fig. 1 we compare the three choices of $a$ in the search for the pasta phases, i.e., $a=0.023 \mathrm{fm}, a=0.1 \mathrm{fm}$, and $a=1 / M_{N}=0.21 \mathrm{fm}$, for $Y_{p}=0.5$. We can see that there is a larger region of the pasta phase for $a=0.023 \mathrm{fm}$. In fact, for $Y_{P}=0.5$ no pasta phases were found with either $a=0.1$ or $a=0.21 \mathrm{fm}$. Therefore, we have chosen $a=0.023 \mathrm{fm}$ to be used throughout our calculations.

It is important to stress that the surface tension coefficient varies with the isospin for a given value of $a$. In Table II, we

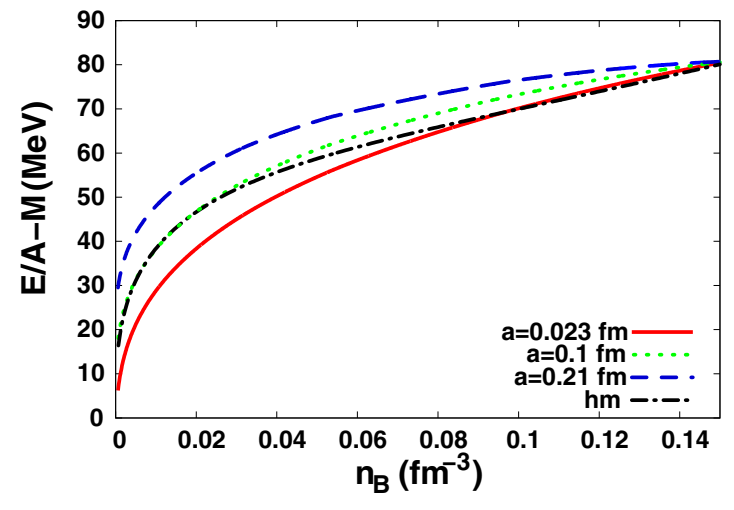

FIG. 1. QMC energy per baryon as a function of the baryon density for proton fraction 0.5 and different choices of $a$. hm stands for homogeneous npe matter.

show the values of $\mathcal{S}$ for seven different proton fractions and $a=0.023$. In order to obtain the pasta phases in $\beta$-equilibrium matter, we have fitted these values of $\mathcal{S}$ to a exponential functional. For the QMC model, a function of the form $\mathcal{S}=d\left(1-e^{-\frac{(x-e)^{2}}{f}}\right)$, with $d=1.58647, e=0.0746702$, and $f=0.265407$ is obtained. For the QMC $\omega \rho$, we have used the same function, with $d=1.41403, e=0.0725015$, and $f=0.206082$. In both cases, $x$ is the global proton fraction $Y_{p}$ and the functions are shown in Fig. 2. We note that there are some works where the proton faction used in the calculation of the surface tension is the one of the denser phase.

Previous works $[1,6,20,26,39,40]$ have shown that the surface tension at zero temperature not only varies with the proton fraction but presents values in between 1.0 and $1.2 \mathrm{MeV} \mathrm{fm}{ }^{-2}$ for $Y_{p}=0.5$ (see Fig. 5 in Ref. [31], for instance). If we constrain the parameter $a$ so that these values are reproduced, we obtain $a=0.020-0.025 \mathrm{fm}$. The value $a=0.020 \mathrm{fm}$ yields $\mathcal{S}=1.0 \mathrm{MeV} \mathrm{fm}^{-2}$ and $a=0.025$ fm results in $\mathcal{S}=1.2 \mathrm{MeV} \mathrm{fm}{ }^{-2}$ for $Y_{p}=0.5$. Choosing $a=0.025$ would increase the surface energy in $8.7 \%$, having a very small effect on the crust-core transition of $\beta$-equilibrium matter. Therefore, we proceed with the comparison of the results obtained with $a=0.020$ and with $a=0.023$, which entails $\mathcal{S}=1.123 \mathrm{MeV} \mathrm{fm}^{-2}$ for $Y_{p}=0.5$. In Table III, we compare the surface tension coefficient $\mathcal{S}$ and the transition density $\rho_{t}$ for the two values of $a$. We see that $\rho_{t}$ is practically

TABLE II. Surface tension coefficient for QMC and QMC $\omega \rho$ models and different proton fractions.

\begin{tabular}{lcc}
\hline \hline & $\begin{array}{c}\text { QMC } \\
Y_{p}\end{array}$ & $\begin{array}{c}\mathrm{QMC} \omega \rho \\
\mathcal{S}\left(\mathrm{MeV} \mathrm{fm}^{-2}\right)\end{array}$ \\
\hline 0.05 & 0.093 & $\mathcal{S}\left(\mathrm{MeV} \mathrm{fm}^{-2}\right)$ \\
0.1 & 0.175 & 0.093 \\
0.2 & 0.393 & 0.194 \\
0.25 & 0.511 & 0.429 \\
0.3 & 0.647 & 0.556 \\
0.4 & 0.923 & 0.692 \\
0.5 & 1.123 & 0.941 \\
\hline \hline
\end{tabular}




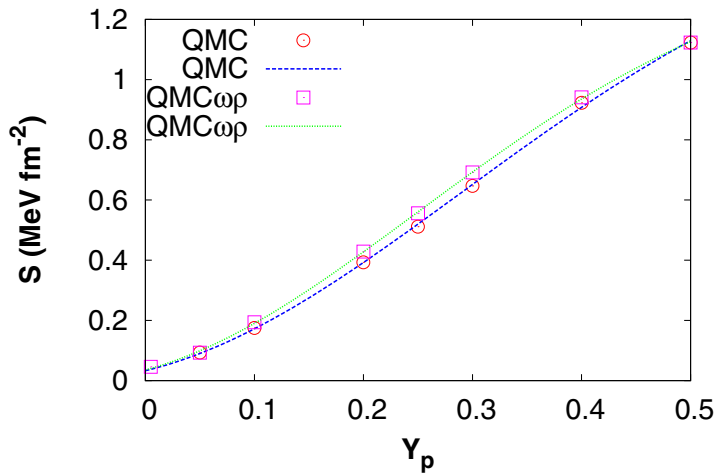

FIG. 2. Surface tension fit for the QMC and QMC $\omega \rho$ models.

independent of $a$ in the range [0.020, 0.023], not only for $\beta$-equilibrium matter but also for matter with fixed proton fractions. The surface tension coefficient as a function of the baryon density is displayed in Fig. 3 for matter in $\beta$ equilibrium, from where we note that $\mathcal{S}$ decreases with the density. We can see that the surface tension coefficient is only slightly larger for $a=0.023 \mathrm{fm}$ both from Table III and Fig. 3 .

\section{RESULTS AND CONCLUSIONS}

Finally, we present our results for the pasta phases obtained with the QMC model at zero temperature, within the coexisting phases approximation. We remark that pasta is only predicted when its free energy per baryon is lower than the homogeneous npe (neutron-proton-electron) matter.

In Fig. 4, we display the free energy per baryon for $Y_{p}=0.5$ and $Y_{p}=0.3$. The curves for $\beta$-equilibrium matter are shown in Fig. 5. The three cases show the presence of pasta phases, which are bigger for larger proton fractions, as already seen in other works. In Fig. 6, we can see the density distribution of the pasta structures. For $Y_{p}=0.5$, three different structures are present: droplets (3D), rods (2D), and slabs (1D), while for $Y_{P}=0.3$, a small amount of tubes (2D) also appear. A similar behavior was obtained in Ref. [44] for different models. The reason was pointed out to the non-self-consistent treatment of the Coulomb force, which prevents a redistribution of protons.

TABLE III. Surface tension coefficient for different proton fractions for the QMC model, $\mathrm{L}=90 \mathrm{MeV}$, and the $a$ values considered. $\rho_{t}$ is the transition density that separates the pasta from the homogeneous phase.

\begin{tabular}{lccc}
\hline \hline$Y_{p}$ & $\begin{array}{c}\mathcal{S} \\
\left(\mathrm{MeV} \mathrm{fm}^{-2}\right)\end{array}$ & $\begin{array}{c}a \\
(\mathrm{fm})\end{array}$ & $\begin{array}{c}\rho_{t} \\
\left(\mathrm{fm}^{-3}\right)\end{array}$ \\
\hline 0.5 & 1.00 & 0.020 & 0.100 \\
0.5 & 1.12 & 0.023 & 0.097 \\
0.3 & 0.58 & 0.020 & 0.094 \\
0.3 & 0.65 & 0.023 & 0.096 \\
0.1 & 0.16 & 0.020 & 0.065 \\
0.1 & 0.17 & 0.023 & 0.058 \\
$\beta$-eq & plot & 0.020 & 0.062 \\
$\beta$-eq & plot & 0.023 & 0.062 \\
\hline \hline
\end{tabular}

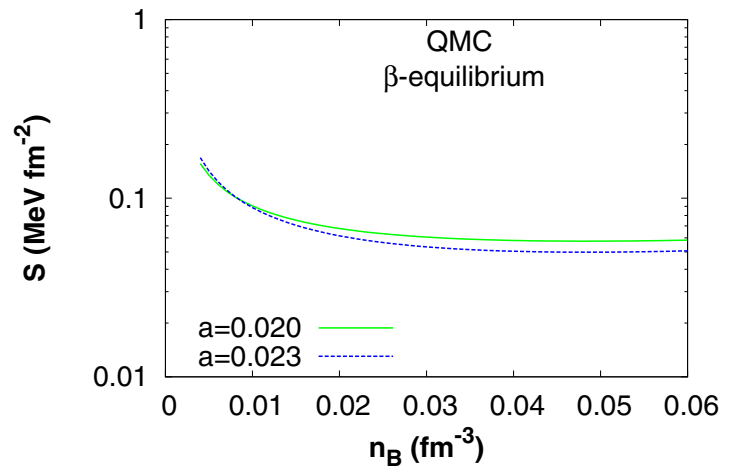

FIG. 3. Surface tension coefficient as a function of the baryon density for $\beta$-equilibrium matter obtained with the QMC model, $L=$ $90 \mathrm{MeV}$, and two choices of $a$.

As a result, the CP method predicts smaller extensions of the pasta phases as a whole and for symmetric matter the larger electron fraction originates stronger Debye screening effects and therefore hinders the appearance of tubes. Althougth the isospin asymmetry affects the structures and distribution of the pasta phases, the difference on the density dependence of the symmetry energy of QMC and QMC $\omega \rho$ is not strong enough to show any effect. In Ref. [45], rods and slabs were present in $\beta$-equilibrium matter; however, the stellar matter
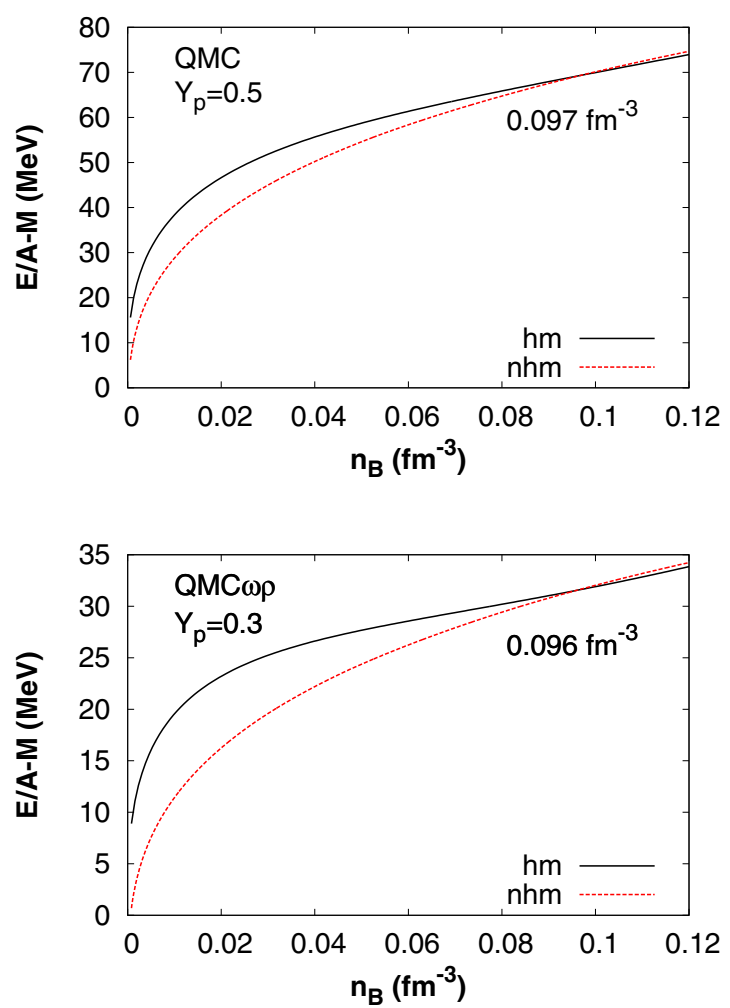

FIG. 4. Free energy per baryon as function of the baryon density calculated with the QMC $\omega \rho$ model and $a=0.023$ for homogenous matter (hm) and nonhomogeneous matter (nhm) with (a) the proton fraction 0.5 and (b) proton fraction 0.3 . 

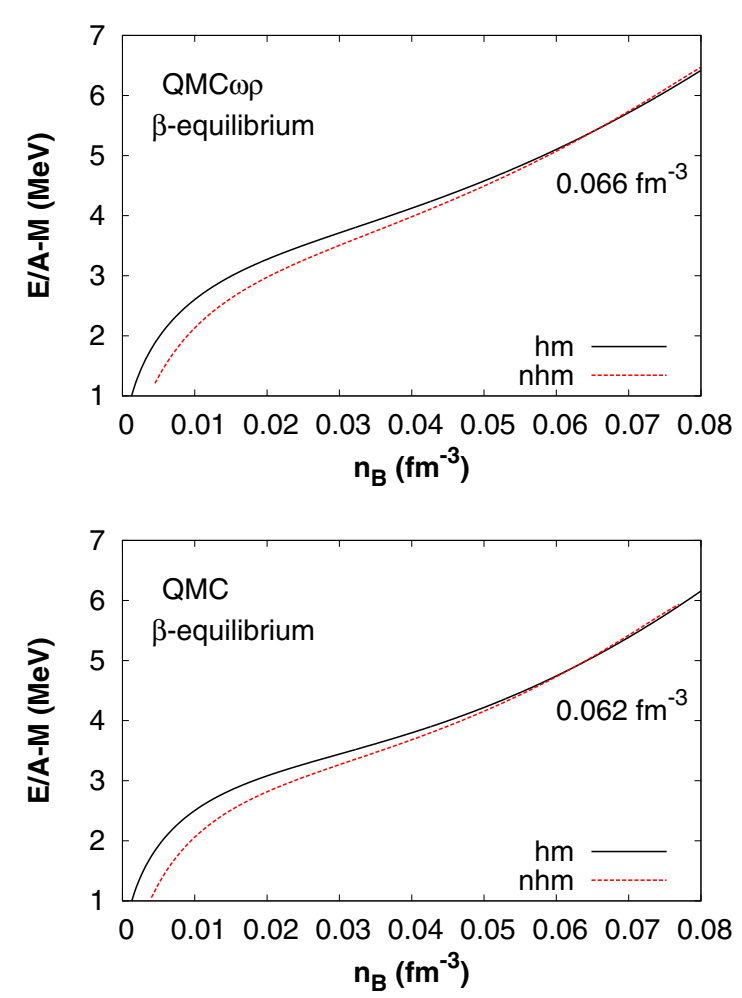

FIG. 5. Free energy per particle for homogeneous (hm) and nonhomogeneous (nhm) matter obtained in $\beta$ equilibrium with QMC $\omega \rho$ with $L=69 \mathrm{MeV}$ (top) and QMC with $L=90 \mathrm{MeV}$ (bottom).

EoS was calculated with models with a smaller slope $L$, in particular, $L \leqslant 60 \mathrm{MeV}$.

The pasta phases shrink with the decrease of the proton fraction and for $\beta$-equilibrium matter only droplets are present. The transition density between the pasta phases and homogeneous matter shows the same behavior as in all models; i.e., it decreases for lower proton fraction and the lowest value is obtained for matter in $\beta$ equilibrium. The calculations performed in Refs. [1] and [20] with the CP method used two different prescriptions for the surface tension coefficient, based

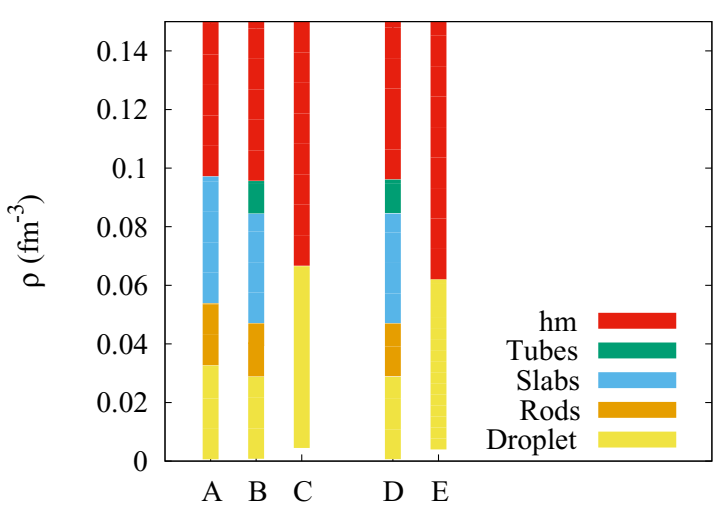

FIG. 6. Phase diagrams at $T=0$ obtained with $\mathrm{CP}$ approximation for QMC $\omega \rho$ with $L=69 \mathrm{MeV}$ : (A) $Y_{p}=0.5$, (B) $Y_{p}=0.3$, (C) $\beta$ equilibrium, and for $\mathrm{QMC}$ with $L=90 \mathrm{MeV}$ : (D) $Y_{p}=0.3$, (E) $\beta$ equilibrium. hm stands for homogeneous matter.

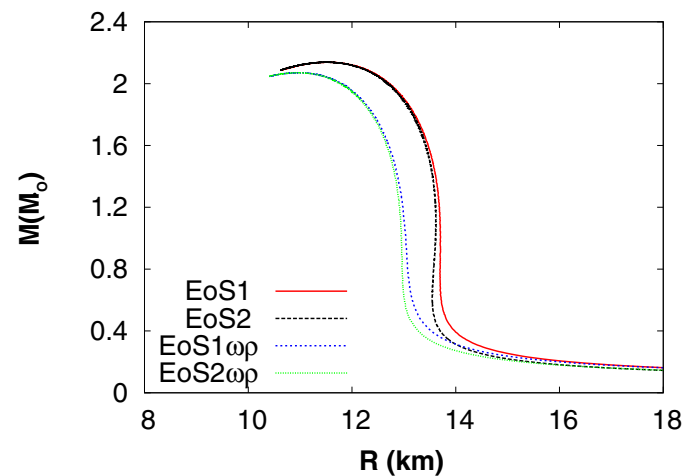

FIG. 7. Mass-radius relation for a family of neutron stars described with the QMC and QMC $\omega \rho$ models with (EoS1 and EoS $1 \omega \rho)$ and without $(\operatorname{EoS} 2$ and $\operatorname{EoS} 2 \omega \rho)$ the pasta phases.

on a fitting of the Thomas-Fermi results to a Skyrme and to relativistic models respectively. Apart from these details in the calculations that can modify slightly the quantitative results, the qualitative conclusions do not differ in general.

We note that in Fig. 5(bottom), the transition core-crust of a neutron star takes place at $n_{B}=0.062 \mathrm{fm}^{-3}$ for the QMC model and at a slightly bigger density, $n_{B}=0.066 \mathrm{fm}^{-3}$, if the $\omega \rho$ interaction is included, as seen in Fig. 5(top). A correlation between the transition densities and the slope has been identified in Refs. [20,46] and in many other works. Since the original QMC model has a symmetry energy slope larger than the QMC $\omega \rho$, a lower crust-core transition density was already expected for that model.

Finally, we analyze the influence of the pasta phases on some neutron star properties. In Fig. 7 we show the mass-radius relation. The $M(R)$ curves are built with four equations of state, from where we can see the influence of the pasta phase and of the $\omega \rho$ interaction on the neutron star properties; see Table IV. EoS 1 and EOS $1 \omega \rho$ contain the pasta phase while EoS2 and EoS2 $\omega \rho$ do not. We have used the homogeneous $\mathrm{QMC}$ and QMC $\omega \rho$ EoS for the core, QMC and QMC $\omega \rho$ with pasta, and the Baym-Bethe-Pethick (BBP) [47] EoS for the inner crust and the Baym-Pethick-Sutherland (BPS) [48] EoS for the outer crust.

If the QMC interaction is considered, EoS1, the BPS + BBP EoS goes up to $n_{B}=3.7 \times 10^{-3} \mathrm{fm}^{-3}$, and the pasta phases lie in between $n_{B}=0.4-6.2 \times 10^{-2} \mathrm{fm}^{-3}$ above this density range the core EoS starts. We match the BPS + BBP EoS directly to the core EoS for densities below $8.9 \times$ $10^{-3} \mathrm{fm}^{-3}$ for EoS2. When the $\omega \rho$ interaction is included,

TABLE IV. Properties of a family of neutron stars obtained with the QMC model. Comparison between equations of state, with and without pasta phase and with and without $\omega \rho$ term.

\begin{tabular}{lccccc}
\hline \hline EoS & Pasta & $L(\mathrm{MeV})$ & $M_{\max }\left(\mathrm{M}_{\odot}\right)$ & $R(\mathrm{~km})$ & $R_{M=1.4 M_{\odot}}(\mathrm{km})$ \\
\hline EoS1 & Yes & 90 & 2.14 & 11.53 & 13.61 \\
EoS $1 \omega \rho$ & Yes & 69 & 2.07 & 10.97 & 12.88 \\
EoS2 & No & 90 & 2.14 & 11.51 & 13.55 \\
EoS2 $\omega \rho$ & No & 69 & 2.07 & 10.96 & 12.83 \\
\hline \hline
\end{tabular}


i.e., for $\mathrm{QMC} \omega \rho, \operatorname{EoS} 1 \omega \rho$, the BPS + BBP EoS goes up to $n_{B}=3.7 \times 10^{-3} \mathrm{fm}^{-3}$, and the pasta phases lie in between $n_{B}=0.45-6.6 \times 10^{-2} \mathrm{fm}^{-3}$ when the core EoS takes on. We match the BPS + BBP EoS directly to the core EoS for densities below $8.9 \times 10^{-3} \mathrm{fm}^{-3}$ for $\operatorname{EoS} 2 \omega \rho$. Note that the maximum masses do not change upon the existence of the pasta phases, and both cases $M_{\max }=2.14 \mathrm{M}_{\odot}(\mathrm{QMC})$ and $M_{\max }=$ $2.07 \mathrm{M}_{\odot}(\mathrm{QMC} \omega \rho)$, satisfy the constraints imposed by the recent measurements of the $2 M_{\odot}$ pulsars PSR J16142230 and PSR J0348+0432 [49,50].

One of the differences between the results obtained with the EoS with and without the pasta phases appears when we compare the radii of typical 1.4-solar-mass neutron stars. The radii when the pasta phase are included is slightly bigger than the radii obtained with the $\mathrm{BPS}+\mathrm{BBP}+$ homogeneous EoS, in accordance with previous findings [26]. All EoS predict radii for a 1.4 $M_{\odot}$ star, inside the radius range proposed in Ref. [51], where the authors constrained the canonical $1.4 \mathrm{M}_{\odot}$ neutron star radii to $R=9.7-13.9 \mathrm{~km}$, or the radius range obtained in Ref. [52] for x-ray bursting NS. However, both are outside the range determined in Ref. [53] from the analysis of spectroscopic radius measurements during thermonuclear bursts or in quiescence or in Ref. [54] from experimental constraints and causality restrictions. The range values proposed in Ref. [51] include the possibility of a phase transition to another form of matter inside the star. If phase transitions are excluded below twice saturation density, the accepted neutron star radii change to $10.7-13.1 \mathrm{~km}$, according to Refs. [55,56]. If this value is accepted, the results obtained with $\operatorname{EoS} 1 \omega \rho$ and $\operatorname{EoS} 2 \omega \rho$, built from the QMC $\omega \rho$ model, are inside the new proposed range. However, the authors of Ref. [57] have made a compilation of neutron star radii estimations from observations for a $1.4 M_{\odot}$ star and have obtained, taking into account a $2 \sigma$ error, values above $14 \mathrm{~km}$. In this case, all models predict radii satisfying this condition.

\section{FINAL REMARKS}

In the present work, we have revisited the calculation of the pasta phases now using a model with quark degrees of freedom, the QMC model. The determination of the inhomogeneous phases was possible by parameterizing the effective nucleon mass at subsaturation density as a nonlinear function of the $\sigma$ meson as already done before in Ref. [36]. Part of the results shown in the present work will take part in a more comprehensive EoS grid that is being built for star cooling and supernova simulations.
Our results depend quantitatively on a parameter necessary for the calculation of the surface tensor coefficient. We have fitted this parameter to the nuclear surface energy and showed that when even changing it in a broad interval the pasta extension was only slightly affected. In other words, the prescription we have used allows us to determine the dependence of the surface energy on the proton fraction, except for the overall normalization that we fix to the usual surface energy.

The general conclusions related to the size of the pasta phases, its internal structure, and the transition density from the pasta to homogeneous matter go in line with the ones obtained in previous works $[1,20]$.

It is important to stress that the value of $L$ obtained with the original QMC parametrization, $L=90 \mathrm{MeV}$, is larger than present constraints impose: The one established in Ref. [55] assumes $63.6 \mathrm{MeV}$ as the largest acceptable value, but larger values are indicated in Ref. [29], where $L<80 \mathrm{MeV}$, and Ref. [30], with $L<86.8 \mathrm{MeV}$, where both studies take into account extra constraints not included in the study of Ref. [55]. To improve the model so that a lower value of $L$ is obtained, we have added a $\omega \rho$ interaction to the Lagrangian density, as already done in previous works for different parametrizations of the nonlinear Walecka model $[33,58]$ and also for the QMC model [32]. This new term in the Lagrangian density changes the density dependence of the symmetry energy and allows the calibration of the model parameters so that a smaller slope of the symmetry energy is obtained at saturation. Our results show that this interaction is mandatory in relativistic mean field models if one wants to conciliate large maximum masses with low radii in neutron stars described by the QMC model.

We intend to incorporate finite-size effects through the implementation of the CLD prescription $[37,38]$ as well. The CLD presents smaller discontinuities at very low densities, so it can be a useful treatment to obtain all the values that will be needed for a complete EoS grid. The inclusion of $\alpha$ particles [39] and other light clusters [40] can also slightly modify the internal structure of the pasta phases.

\section{ACKNOWLEDGMENTS}

D.P.M. (Grant No. 300602/2009-0) and G.G. (doctorate scholarship) acknowledge support from $\mathrm{CNPq}$ and Capes. C.P. acknowledges partial support from FCT (Portugal) under project UID/FIS/04564/2016, and by "NewCompStar" COST Action MP1304.
[1] S. S. Avancini, D. P. Menezes, M. D. Alloy, J. R. Marinelli, M. M. W. Moraes, and C. Providência, Phys. Rev. C 78, 015802 (2008).

[2] J. Xu, L. Chen, B. Li, and H. Ma, Phys. Rev. C 79, 035802 (2009).

[3] D. G. Ravenhall, C. J. Pethick, and J. R. Wilson, Phys. Rev. Lett. 50, 2066 (1983).
[4] C. J. Horowitz, M. A. Pérez-García, and J. Piekarewicz, Phys. Rev. C 69, 045804 (2004).

[5] C. J. Horowitz, M. A. Pérez-García, D. K. Berry, and J. Piekarewicz, Phys. Rev. C 72, 035801 (2005).

[6] T. Maruyama, T. Tatsumi, D. N. Voskresensky, T. Tanigawa, and S. Chiba, Phys. Rev. C 72, 015802 (2005).

[7] C. J. Pethick and A. Y. Potekhin, Phys. Lett. B 427, 7 (1998). 
[8] C. Bertulani and J. Piekarewicz, Neutron Star Crust (Nova Science Publishers, New York, 2012), p. 337.

[9] B. Link, R. I. Epstein, and J. M. Lattimer, Phys. Rev. Lett. 83, 3362 (1999).

[10] M. D. Alloy and D. P. Menezes, Phys. Rev. C 83, 035803 (2011).

[11] U. Furtado, S. Avancini, J. Marinelli, W. Martarello, and C. Providência, Eur. Phys. J. A 52, 290 (2016).

[12] H. Shen, H. Toki, K. Oyamatsu, and K. Sumiyoshi, Nucl. Phys. A 637, 435 (1998).

[13] H. Shen, H. Toki, K. Oyamatsu, and K. Sumiyoshi, Astrophys. J. Suppl. 197, 20 (2011).

[14] M. Hempel, T. Fischer, J. Schaffner-Bielich, and M. Liebendörfer, Astrophys. J. 748, 70 (2012).

[15] A. W. Steiner, M. Hempel, and T. Fischer, Astrophys. J. 774, 17 (2013).

[16] S. Banik, M. Hempel, and D. Bandyopadhyay, Astrophys. J. Suppl. Ser. 214, 22 (2014).

[17] J. M. Lattimer and F. D. Swesty, Nucl. Phys. A 535, 331 (1991).

[18] H. Sonoda, G. Watanabe, K. Sato, T. Takiwaki, K. Yasuoka, and T. Ebisuzaki, Phys. Rev. C 75, 042801(R) (2007).

[19] H. Sonoda, G. Watanabe, K. Sato, K. Yasuoka, and T. Ebisuzaki, Phys. Rev. C 77, 035806 (2008).

[20] S. S. Avancini, L. Brito, J. R. Marinelli, D. P. Menezes, M. M. W. de Moraes, C. Providência, and A. M. Santos, Phys. Rev. C 79, 035804 (2009).

[21] G. Watanabe, H. Sonoda, T. Maruyama, K. Sato, K. Yasuoka, and T. Ebisuzaki, Phys. Rev. Lett. 103, 121101 (2009).

[22] H. Pais and J. R. Stone, Phys. Rev. Lett. 109, 151101 (2012).

[23] P. A. M. Guichon, Phys. Lett. B 200, 235 (1988).

[24] K. Saito and A. W. Thomas, Phys. Lett. B 327, 9 (1994).

[25] K. Saito and A. W. Thomas, Phys. Rev. C 52, 2789 (1995).

[26] H. Pais, D. P. Menezes, and C. Providência, Phys. Rev. C 93, 065805 (2016).

[27] G. Grams, A. M. Santos, and D. P. Menezes, Braz. J. Phys. 46, 111 (2016).

[28] A. M. Santos, C. Providência, and P. K. Panda, Phys. Rev. C 79, 045805 (2009).

[29] M. Dutra, O. Lourenço, S. S. Avancini, B. V. Carlson, A. Delfino, D. P. Menezes, C. Providência, S. Typel, and J. R. Stone, Phys. Rev. C 90, 055203 (2014).

[30] M. Oertel, M. Hempel, T. Klähr, and S. Typel, Rev. Mod. Phys. 89, 015007 (2017).

[31] C. Providência, S. S. Avancini, R. Cavagnoli, S. Chiacchiera, C. Ducoin, F. Grill, J. Margueron, D. P. Menezes, A.Rabhi, I. Vidaña, Eur. Phys. J. A 50, 44 (2014).

[32] P. K. Panda, A. M. S. Santos, D. P. Menezes, and C. Providência, Phys. Rev. C 85, 055802 (2012).
[33] R. Cavagnoli, D. P. Menezes, and C. Providência, Phys. Rev. C 84, 065810 (2011).

[34] N. Barik, R. N. Mishra, D. K. Mohanty, P. K. Panda, and T. Frederico, Phys. Rev. C 88, 015206 (2013).

[35] R. N. Mishra, H. S. Sahoo, P. K. Panda, N. Barik, and T. Frederico, Phys. Rev. C 92, 045203 (2015).

[36] P. A.M. Guichon, K. Saito, E. Rodionov, and A. W. Thomas, Nucl. Phys. A 601, 349 (1996).

[37] S. S. Bao, J. N. Hu, Z. W. Zhang, and H. Shen, Phys. Rev. C 90 , 045802 (2014).

[38] H. Pais, S. Chiacchiera, and C. Providência, Phys. Rev. C 91, 055801 (2015).

[39] S. S. Avancini, C. C. Barros, Jr., D. P. Menezes, and C. Providência, Phys. Rev. C 82, 025808 (2010).

[40] S. S. Avancini, C. C. Barros, L. Brito, S. Chiacchiera, D. P. Menezes, and C. Providência, Phys. Rev. C 85, 035806 (2012).

[41] J. Randrup, Phys. Rev. C 79, 054911 (2009).

[42] M. B. Pinto, V. Koch, and J. Randrup, Phys. Rev. C 86, 025203 (2012).

[43] G. A. Lalazissis, J. König, and P. Ring, Phys. Rev. C 55, 540 (1997).

[44] S. S. Avancini, S. Chiacchiera, D. P. Menezes, and C. Providência, Phys. Rev. C 82, 055807 (2010).

[45] F. Grill, C. Providência, and S. S. Avancini, Phys. Rev. C 85, 055808 (2012).

[46] I. Vidaña, C. Providência, A. Polls, and A. Rios, Phys. Rev. C 80, 045806 (2009).

[47] G. Baym, H. A. Bethe, and C. J. Pethick, Nucl. Phys. A 175, 225 (1971).

[48] G. Baym, C. Pethick, and P. Sutherland, Astrophys. J. 170, 299 (1971)

[49] P. B. Demorest, T. Pennucci, S. M. Ransom, M. S. E. Roberts, and J. W. T. Hessels, Nature (London) 467, 1081 (2010).

[50] J. Antoniadis et al., Science 340, 1233232 (2013).

[51] K. Hebeler, J. M. Lattimer, C. J. Pethick, and A. Schwenk, Phys. Rev. Lett. 105, 161102 (2010).

[52] V. F. Suleimanov, J. Poutanen, D. Klochkov, and K. Wener, Eur. Phys. J. A 52, 20 (2016).

[53] F. Özel, D. Psaltis, T. Güver, G. Baym, C. Heinke, and S. Guillot, Astrophys. J. 820, 28 (2016).

[54] A. W. Steiner, J. M. Lattimer, and E. Brown, Eur. Phys. J. 52, 18 (2016).

[55] J. M. Lattimer and Y. Lim, Astrophys. J. 771, 51 (2013).

[56] J. M. Lattimer and M. Prakash, Phys. Rep. 621, 127 (2016).

[57] M. Fortin, J. L. Zdunik, P. Haensel, and M. Bejger, Astron. Astrophys. 576, A68 (2015).

[58] C. J. Horowitz and J. Piekarewicz, Phys. Rev. Lett. 86, 5647 (2001). 